\title{
Flying through the coronary easily
}

\author{
Francesco Prati, Mario Albertucci \\ San Giovanni Hospital and CLI Foundation, Rome, Italy
}

Article, p. 574

Optical coherence tomography (OCT) has been waived as a breakthrough in coronary imaging. With its superb resolution in the range of $10-20 \mu \mathrm{m}$, OCT has proven to be a valuable technique in guiding coronary interventions [1-3]. Apart from

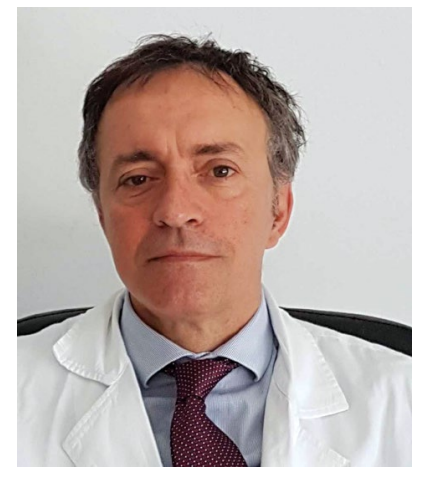

the most troublesome complications after coronary interventions. The fact that OCT requires additional contrast use, certainly acts as an obstacle to its widespread utilization. Although in the present high volume OCT center it is applied as an established methodology for OCT acquisition, requires the same contrast volume, regardless of patient characteristics, and of the coronary this, among other intracoronary technical solutions, OCT certainly has the greatest potential in studying atherosclerosis in vivo, and identifying subjects with vulnerable lesions which leave patients prone to developing hard coronary events.

Among the steps that enabled OCT to gain such credibility, the adoption of the non-occlusive techniques to acquire images has certainly played an instrumental role $[4,5]$. Subsequently, the development of frequency domain technology, capable of acquiring long segments in a few seconds, and because of this high speed, it has made OCT a simple, user-friendly technique.

However, even in skilled hands, OCT acquisition may be suboptimal, and the need for an increased amount of contrast remains a reason for concern. For this reason, the paper by Gutiérrez-Chico et al. [6] published in this number of "Cardiology Journal" is timely, and of great interest. Authors developed an interesting concept, showing that the application of a simple formula, taking into account the pullback speed of OCT and the length of segment of interest, can reduce the amount of contrast and improve image quality. The latter remains a problem particularly for accurate plaque characterization, where features of vulnerability such as measurements of fibrous cap thickness or identification of macrophage requires a resolution slightly higher than cellular. On the other hand, renal insufficiency is one of segment to be visualized. It is felt herein, that a personalized adoption of OCT acquisitions should be implemented. Having said this, it does follow the concept of personalized medicine which has been popularized over the last few years, where every patient requires dedicated treatment.

Professor Juan Luis Gutiérrez-Chico and coauthors should be congratulated for what they have done. There is now a further step to be done. To better understand the clinical impact and ease of use in this innovative solution in acquiring OCT images, there is now need to test it in other centres exactly as it was done over 10 years ago for the non-occlusive technique.

Conflict of interest: With Abbott as consultant.

\section{References}

1. Prati F, Regar E, Mintz GS, et al. Expert review document on methodology, terminology, and clinical applications of optical coherence tomography: physical principles, methodology of image acquisition, and clinical application for assessment of coronary arteries and atherosclerosis. Eur Heart J. 2010; 31(4): 401-415, doi: 10.1093/eurheartj/ehp433, indexed in Pubmed: 19892716.

2. Prati F, Guagliumi G, Mintz GS, et al. Expert's OCT Review Document. Expert review document part 2: methodology, terminology and clinical applications of optical coherence tomography for the assessment of interventional procedures. Eur Heart J. 2012; 33(20): 2513-2520, doi: 10.1093/eurheartj/ehs095, indexed in Pubmed: 22653335.

Address for correspondence: Prof. Francesco Prati, San Giovanni Hospital and CLI Foundation, Rome, Italy, e-mail: fprati61@gmail.com 
Cardiology Journal 2018, Vol. 25, No. 5

3. Gutiérrez-Chico JL, Alegría-Barrero E, Teijeiro-Mestre R, et al. Optical coherence tomography: from research to practice. Eur Heart J Cardiovasc Imaging. 2012; 13(5): 370-384, doi: 10.1093/ /ehjci/jes025, indexed in Pubmed: 22330231.

4. Prati F, Cera M, Ramazzotti V, et al. Safety and feasibility of a new non-occlusive technique for facilitated intracoronary optical coherence tomography (OCT) acquisition in various clinical and anatomical scenarios. EuroIntervention. 2007; 3(3): 365-370, indexed in Pubmed: 19737719.
5. Prati F, Cera M, Ramazzotti V, et al. From bench to bedside: a novel technique of acquiring OCT images. Circ J. 2008; 72(5): 839-843, indexed in Pubmed: 18441468.

6. Gutiérrez-Chico JL, Cortés C, Schincariol M, et al. A formula to calculate the contrast volume required for optimal imaging quality in optical coherence tomography with non-occlusive technique. Cardiol J. 2018; 25(5): 574-581, doi: 10.5603/ /CJ.a2018.0112, indexed in Pubmed: 30246237. 